\title{
Perspective on treatment for unresectable locally advanced non-small cell lung cancer with oncogene-driven mutation: a narrative review
}

\author{
Liyang Jiang, Xiangjiao Meng, Xianguang Zhao, Ligang Xing, Jinming Yu \\ Department of Radiation Oncology, Shandong Cancer Hospital and Institute, Shandong First Medical University and Shandong Academy of \\ Medical Sciences, Jinan, China \\ Contributions: (I) Conception and design: L Xing; (II) Administrative support: L Xing, J Yu; (III) Provision of study materials or patients: None; (IV) \\ Collection and assembly of data: L Jiang, X Meng; (V) Data analysis and interpretation: L Jiang, X Meng, X Zhao, L Xing; (VI) Manuscript writing: \\ All authors; (VII) Final approval of manuscript: All authors. \\ Correspondence to: Ligang Xing, MD. Department of Radiation Oncology, Shandong Cancer Hospital and Institute, Shandong First Medical \\ University and Shandong Academy of Medical Sciences, 440 Jiyan Road, Jinan, China. Email: xinglg@medmail.com.cn.
}

\begin{abstract}
The standard treatment of unresectable locally advanced non-small cell lung cancer (LA NSCLC) is concurrent chemoradiotherapy. With the addition of immunotherapy, patients with LA NSCLC received a significantly prolonged outcome, while patients with harboring epidermal growth factor receptor (EGFR) mutation benefited less. Tyrosine kinase inhibitors (TKIs) have revolutionized the treatment of stage IV with harboring EGFR mutation and anaplastic lymphoma kinase rearrangement, but there are few recommendations indicating whether TKI treatment is effective in unresectable NSCLC. Preclinical studies have shown that TKIs could have a radiosensitizing effect, which provided a rationale to consider the application TKI with radiotherapy. In this review, we summarize the clinical studies that have used TKIs in LA-NSCLC as well as ongoing trials, and discuss recent progress in research related to the efficacy of TKI for unresectable LA NSCLC patients. Recent results of small studies evaluating TKI therapy for LA NSCLC patients in combination with radiation or chemoradiation demonstrated promising efficacy, improved outcomes with a tolerable toxicity profile. However, there is a lack of strong evidence for TKI treatment in unresectable LA NSCLC, because of unpowered statistics, lack of molecular selection, or lack of large randomized arms. We prospect the combination of TKI and radiation or chemoradiation therapy might eventually replace the current standard treatment for patients with LA NSCLC harboring oncogenedriven mutation.
\end{abstract}

Keywords: Non-small cell lung cancer (NSCLC); locally advanced stage (LA stage); epidermal growth factor receptor (EGFR); anaplastic lymphoma kinase (ALK); targeted therapy

Submitted Mar 27, 2020. Accepted for publication Aug 10, 2020.

doi: $10.21037 /$ tlcr-20-722

View this article at: http://dx.doi.org/10.21037/tlcr-20-722

\section{Introduction}

Lung cancer is the leading cause of cancer-related death worldwide, accounting for 1.8 million deaths each year (1). Non-small cell lung cancer (NSCLC) represents $85 \%$ of all primary lung cancers (2). About $20 \%$ to $25 \%$ of NSCLC patients are diagnosed with locally advanced (LA) disease (3). For unresectable LA NSCLC patients with good performance status, concurrent chemoradiotherapy (CCRT) is currently recommended as standard treatment with 5-year overall survival (OS) rates between 15-32\% (4-6). Unfortunately, the survival outcomes of these patients seem to reach a plateau despite the use of newer chemotherapy agents, therefore, the optimal concurrent chemotherapy regimen has not yet been determined (7). The RTOG 


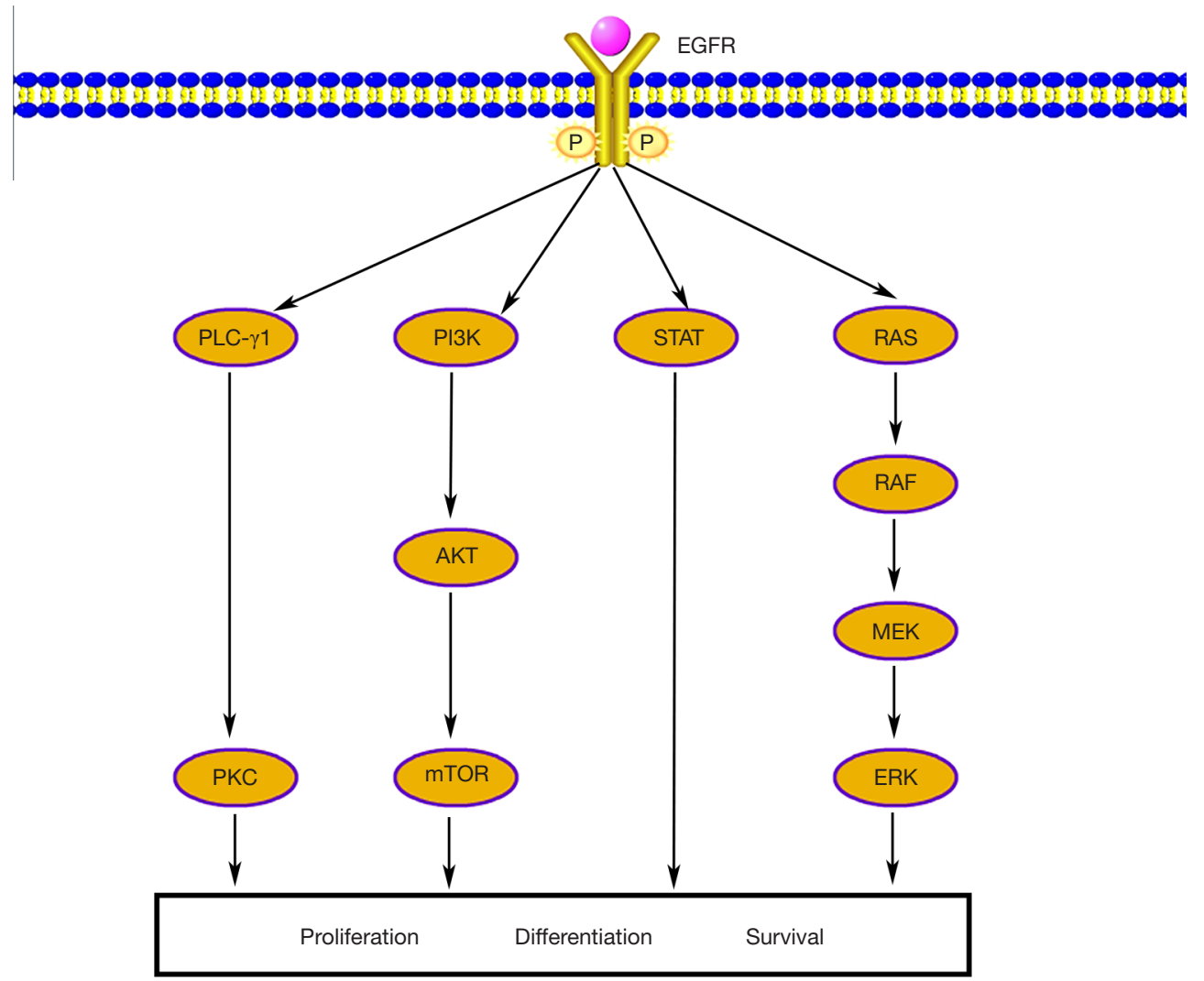

Figure 1 Simplified signaling pathways activated by epidermal growth factor receptor.

0617 trial concluded the addition of cetuximab did not improve survival, and the standard radiation dose of $60 \mathrm{~Gy}$ was still better than 74 Gy (6). Moreover, consolidation chemotherapy is not recommended after standard CCRT due to no prognosis improvement of LA-NSCLC (8). In the phase III PACIFIC trial, treatment with durvalumab, a programmed cell death-ligand 1 inhibitor, significantly prolonged progression-free survival (PFS) in patients with LA NSCLC (9). However, patients with the epidermal growth factor receptor (EGFR) mutation probably benefited less from this treatment.

EGFR is a transmembrane glycoprotein that constitutes one of four members of the ErbB family of tyrosine kinase receptors. Binding EGFR to its ligands leads to autophosphorylation of receptor tyrosine kinase and subsequent activation of signal transduction pathways that are involved in regulating cellular proliferation, differentiation and survival (Figure 1) (10).

Tyrosine kinase inhibitors (TKIs) have revolutionized the treatment of stage IV NSCLC with harboring
EGFR mutation and anaplastic lymphoma kinase (ALK) rearrangement. First-generation EGFR- and ALK-TKIs such as gefitinib, erlotinib, and crizotinib have been shown to significantly prolong PFS and double the overall response rate as compared with platinum-doublet chemotherapy (11-13). Meanwhile, a third-generation EGFR-TKI, osimertinib, has demonstrated improved PFS and even OS, over the previous generation of TKIs $(14,15)$. Although these clinical trials included stage IIIB and stage IV patients, the proportion of stage IIIB patients was low.

Some studies suggest that the proportion of LA NSCLC harboring EGFR mutation is about 10-30\% with the ALK rearrangement proportion being about 2-8\% (16-18). Further studies discovered that, with the treatment of chemoradiotherapy (CRT), the PFS of these patients was significantly poorer than the wild-type EGFR patients, while the frequency of distant metastasis was higher (1921). Meanwhile, other research has reported a better OS rate in a EGFR-mutant group compared with that of a wild-type group, but the difference was not statistically 
significant $(19,22)$.

Thus, what treatment constitutes the best opinion for unresectable LA NSCLC oncogene-harboring driven mutation remains unclear. It is possible that the that positive results for stage IV patients might extend to those with LA NSCLC. We here summarize the recent progress in research related to the efficacy of TKIs for unresectable LA NSCLC patients as well as ongoing trials including with ALK rearrangement. We present the following article in accordance with the Narrative Review Checklist (available at http://dx.doi.org/10.21037/tlcr-20-722).

\section{EGFR-TKIs alone}

A retrospective study compared the outcomes of TKIs and CCRT for stage IIIB lung adenocarcinoma patients with EFGR mutation (23). Hsia et al. collected the treatment information of 177 TKIs and 22 CCRT patients from 2011 to 2015 , yielding a 5 -year OS rates in the TKIs and CCRT group of approximately $30 \%$ and $26 \%$, respectively, but no statistically significant differences between the groups. Given the limited data, a definite conclusion cannot be drawn concerning whether the application of EGFRTKIs alone is a preferred treatment for EGFR-mutant LA NSCLC.

\section{EGFR-TKIs and radiotherapy}

Preclinical studies have shown that EGFR-mutant NSCLC cells have a predominantly radiosensitive phenotype, with gefitinib being capable of radiosensitizing tumor cells and erlotinib being capable of enhancing the cytotoxic effects of radiation, suggests that the TKIs could have a radiosensitizing effect (24-26). Several mechanisms have been identified in preclinical studies that can help improve local tumour control when treated with radiation plus TKIs. These include direct kill of cancer stem cells by TKIs, cellular radiosensitization through modified signal transduction, inhibition of repair of DNA damage, reduced repopulation and improved reoxygenation during fractionated radiotherapy (27). These facts provide a rationale to consider the application EGFR-TKIs with concurrent radiotherapy. In unselected patients, a series of 9 unresectable stage III NSCLC patients were treated with a 2-week induction of gefitinib followed by gefitinib with concurrent definitive radiotherapy (28). The high incidence of pulmonary toxicity led to closure of the study, with only 4 patients completing the planned treatment and experiencing partial responses; 3 of these 4 patients lived over 60 months without local recurrence, while only 2 them exhibited the sensitizing EFGR mutation, with both surviving over 5 years. Furthermore, no more unexpected toxicity occurred during the treatment with gefitinib and radiotherapy. Additionally, Rothschild et al.'s study also confirmed the feasibility and tolerability of gefitinib in combination with definitely radiotherapy (29): no lung toxicity was found in 5 patients and adverse events were grade 1-2 skin and subcutaneous tissue toxicities.

RECEL (NCT01714908) was a randomized phase II trial, that aimed to compare erlotinib with radiotherapy versus etoposide-cisplatin with radiotherapy for EGFRmutated LA NSCLC. A total of 252 patients were screened, and 41 were eventually enrolled into 2 arms. Early results revealed that the erlotinib and radiotherapy arm had significantly improved PFS as compared to the CRT arm (27.86 vs. 6.41 months, hazard ratio $0.053,95 \%$, confidence interval $0.006-0.463, \mathrm{P}<0.001)$ with same incidence of adverse effects being found in the 2 arms (Common Terminology Criteria for Adverse Events grade $\geq 1,86.7 \%$, $13 / 15)$. The most common grade $\geq 3$ severe adverse effects were rash $(20 \%, 3 / 15)$ and hematological toxicity $(26.7 \%$, 4/15) (30). Data is still being collected from the ongoing phase II WJOG6911L trial which is analyzing gefitinib with concurrent radiation ( $64 \mathrm{~Gy} / 32 \mathrm{~F}$ ) followed by gefitinib maintenance for up to 2 years in 27 patients with EGFRmutated unresectable LA NSCLC (31). For the EGFRmutated LA NSCLC patients, radiotherapy and the addition of EGFR-TKIs, rather than chemotherapy, have yielded positive and promising survival results.

\section{EGFR-TKIs and CRT}

In the early 2000s, EGFR-TKIs were evaluated in concurrent and maintenance settings in LA NSCLC. A phase I trial of erlotinib combined with CCRT failed to demonstrate the benefit of TKI addition (32). Patients received erlotinib only during CCRT. The survival results were disappointing in the unselected patients, and those with EGFR mutation also showed no significant OS difference. However, erlotinib was well tolerated and no additional radiation pneumonitis was observed. The SWOG S0023 trial also failed to prove any survival benefit (33). This phase III trial randomized 620 unselected LA NSCLC patients after CCRT and docetaxel consolidation to receive up to 5 years of gefitinib or placebo. Patients receiving gefitinib had unexpectedly decreased survival 
compared with those receiving placebo (23 vs. 35 months, $\mathrm{P}=0.013)$. The gefitinib was well-tolerated and thus the disappointing survival results might not have resulted from treatment toxicity; rather, the poor outcome was ascribed to the lack of oncogene selection in patients. The CALGB 30106 trial randomized 60 unselected patients to receive 2 cycles of induction paclitaxel-carboplatin chemotherapy with concurrent gefitinib followed by gefitinib and definitive radiotherapy ( $66 \mathrm{~Gy} / 33 \mathrm{~F}$ ) in poor-risk patients (performance status 2 or weight loss $\geq 5 \%$ ) or gefitinib and definitive radiotherapy plus weekly paclitaxel-carboplatin chemotherapy in good-risk patients (performance status $0-1$ or weight loss $<5 \%$ ), and gefitinib maintenance (34). Similar to the result of the SWOG S0023 trial, the survival of good-risk patients receiving CCRT plus gefitinib was disappointing and even worse than that of the poor-risk patients (13 vs. 19 months). Furthermore, no difference was found in median OS and PFS between the EGFR-mutated patients and the wild-type patients (8.5 vs. 15.3 months, $\mathrm{P}=0.8834$; 6.7 vs. 11.4 months, $\mathrm{P}=0.8778$ ' respectively). Compared with historical data, acute high-grade infield toxicity was not increased.

The CALGB 30605/RTOG 0972 trial focused on poorrisk patients who do not seem to benefit from standard CRT treatment (35). In the previous CALGB 30106 trial, poor-risk patients were defined as performance status 2 or weight loss $\geq 5 \%$, and the median survival reached an unprecedented 19 months (34). The CALGB 30605/ RTOG 0972 trial enrolled poor-risk patients who had either performance status 2 or performance status $0-1$ and $\geq 10 \%$ weight loss within 3 months. They received 2 cycles of induction nab-paclitaxel-carboplatin chemotherapy followed by erlotinib concurrently with radiotherapy (66 Gy/33 F). Molecular data were available for 31 out of 75 patients. Unexpectedly, no EGFR-mutated patients were identified. Treatment-related adverse events were well tolerated. Grade 3 esophagitis was observed in 4 patients and pneumonitis in only 1 patient. The median PFS and OS were 11 and 17 months, respectively. The overall 1-year OS was $57 \%$, which narrowly missed the prespecified target for significance.

The JCOG 0402 trial, however, achieved somewhat encouraging survival results, with a $73 \%$ objective response rate, 28.5 months median OS, and $65.4 \%$ 2-year OS (36). In this trial, 38 unselected patients received 2 cycles of induction vinorelbine-cisplatin chemotherapy followed by gefitinib (up to 1 year) and concurrent radiotherapy (60 Gy/30 F). Similar to the SWOG S0023 trial, gefitinib maintenance did not also increase the rate of pneumonitis. Erlotinib also obtained promising survival results in a phase II trial reported by Komaki et al. (37). In this trial, 46 unselected patients received 7 weekly cycles of paclitaxel-carboplatin chemotherapy (every Monday) concurrent radiation (every Monday through Friday, 63 Gy/35 F) and erlotinib (every Tuesday through Sunday), followed by 2 cycles of paclitaxelcarboplatin consolidation chemotherapy after a 1-month break. Although median PFS time was 14.0 months (failing to meet authors' hypothesis of 15 to 25 months), the median OS and 2-year OS satisfactorily reached 36.5 months and $67.4 \%$, respectively. These findings may suggest a potential survival benefit of TKIs, although the EGFR status was either unknown or wild type in all trials.

As it was realized that EGFR-TKIs were becoming more effective in patients with EGFR mutation, subsequent studies more rationally designed the treatment regimens according to EGFR mutation status. In a single-arm study, 62 EGFR-mutated stage III-IV patients received 4 cycles of platinum-based doublet chemotherapy followed by radiotherapy $(60 \mathrm{~Gy} / 30 \mathrm{~F})$ concurrent erlotinib, and erlotinib maintenance (38). Of the 62 enrolled patients, 37 were in stage III and 12 were in stage IV. Median PFS was 7.4 months, and OS was 12.9 months for stage III disease, with tolerable toxicity. Lee et al. conducted 2 parallel randomized phase II studies depending on EGFR mutation status (39). EGFR-mutated patients received 3 cycles of erlotinib first and were randomized to either erlotinib with concurrent radiation $(60 \mathrm{~Gy} / 30 \mathrm{~F})$ followed by erlotinib for consolidation for 6 cycles or just CCRT with irinotecancisplatin. The EGFR unknown or wild-type patients were randomized to receive either 3 cycles of irinotecancisplatin before or after CCRT with irinotecan-cisplatin. Longer survival was observed in EGFR-mutated patients, with an excellent 74.8-month median OS, compared with a 25.3-month OS in EGFR wild-type patients $(\mathrm{P}=0.034)$. Brain metastasis was more common as the first relapse site in the EGFR-mutated patients compared to those with the EGFR wild-type or unknown mutations, which could may indicate that the addition of EGFR-TKI could better control the extracranial disease.

The ongoing phase II LOGIK0902/OLCSG0905 trial is aimed at analyzing the survival results of 8 -week gefitinib followed by docetaxel-cisplatin concurrent radiotherapy $(60 \mathrm{~Gy} / 30 \mathrm{~F})$ in 21 patients with unresectable LA NSCLC harboring EGFR mutation (40). The ongoing phase II RTOG 1306 (NCT01822496) trial has randomized EGFRmutated patients to receive either erlotinib induction 
Table 1 Study characteristics

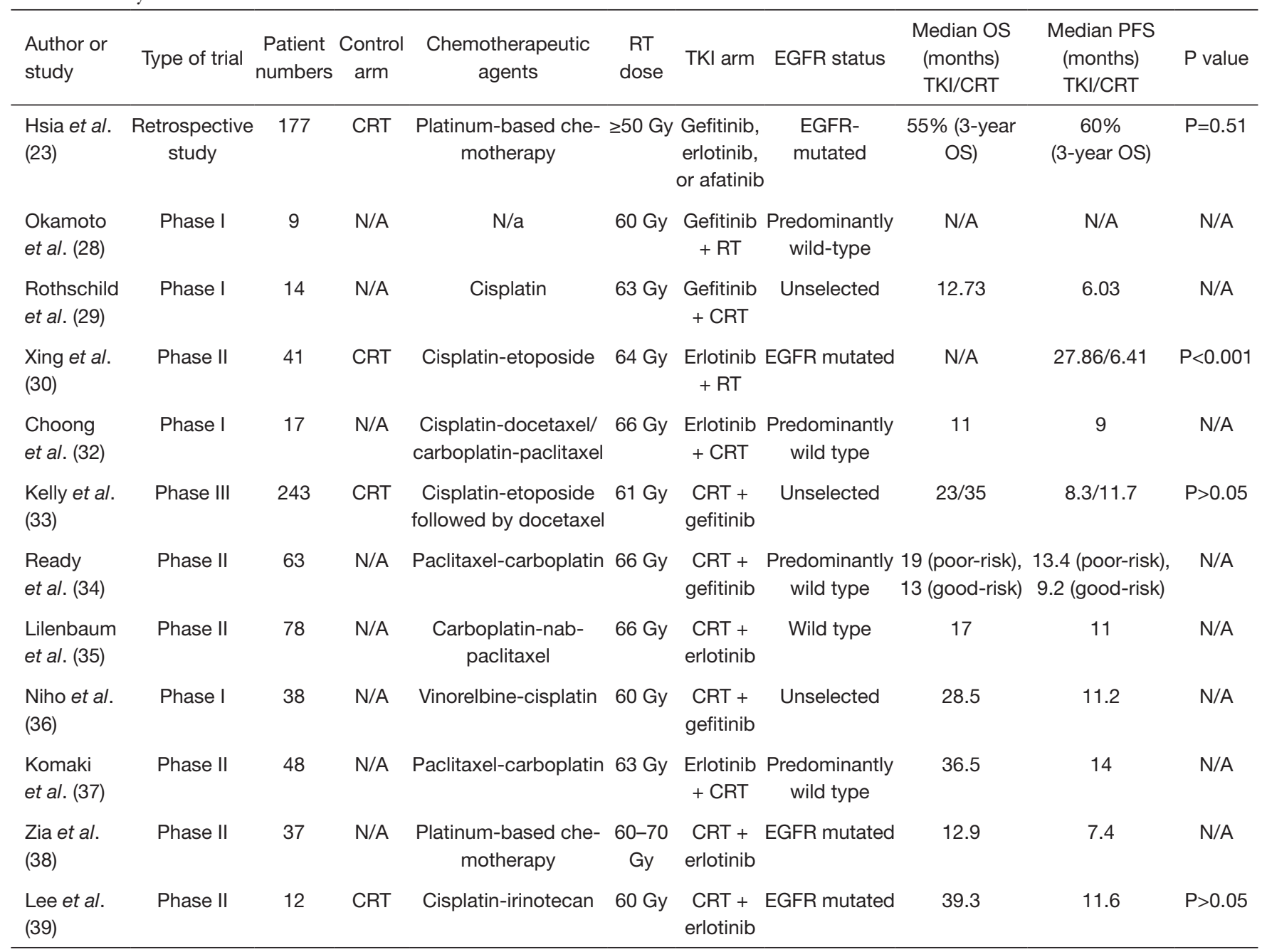

CRT, chemoradiotherapy; EGFR, epidermal growth factor receptor; RT, radiotherapy; N/A, not available; TKI, tyrosine kinase inhibitor; OS, overall survival; PFS, progress-free survival.

for 3 months followed by CCRT or CCRT only. ALKrearrangement patients have also been randomized in this trial and are described in the next section. The phase III LAURA trial (NCT03521154) will evaluate CCRT followed by osimertinib consolidation in EGFR-mutated unresectable LA NSCLC, while the ongoing observational NCT04304638 trial is exploring the survival differences of 3 treatment strategies (CRT, radiation plus EGFR-TKI, and EGFR-TKI only) based on the real-world data.

\section{ALK-TKIs \& CRT}

Thus far, only 1 study protocol has begun to explore the treatment of unresectable LA-NSCLC harboring ALK rearrangement. As mentioned above, the ongoing phase II RTOG 1306 (NCT01822496) has also randomized ALKpositive patients to receive either crizotinib induction for 3 months followed by chemotherapy (either cisplatin/ etoposide or paclitaxel/carboplatin) plus concurrent radiotherapy (60 Gy/30 F) or CCRT only. The primary outcome measure is PFS, and the final results are highly anticipated.

The data and characteristics from all the published studies mentioned in this review are summarized in Table 1 .

\section{Conclusions}

Currently, the use of TKIs is not recommended in 
any practice guidelines or expert consensus for LANSCLC patients $(41,42)$. The available data thus far has demonstrated potential benefit from TKIs in EGFRmutated unresectable LA-NSCLC patient, with no data available for in ALK-positive patients. However, given the lack of strong supporting data, large randomized trials with sufficiently powered arms, accurate patient selection, appropriate duration of TKI therapy, and effective control arms are needed to test whether the benefit of TKIs shown in advanced NSCLC is transferrable to LA-NSCLC patients, in the same way that endocrine therapy has been applied in breast cancer. The addition of TKIs treatment indeed bring about the incidence of adverse events includes rash, esophagitis, pneumonitis and hematological toxicity, but they can be tolerated. For patients safety considerations, we think that patients with good pulmonary function and good condition may benefit more from TKIs treatment. But questions concerning if and when to use chemotherapeutic agents, appropriate radiation dose and volume, and the timing and modality of TKI treatment need to be solved in the future. With the advent of the era of immunotherapy, NSCLC patients have gained considerable survival improvement, but those patients with EGFR- or ALKpositive mutations have seen little benefit (43). Similar to immunotherapy, pneumonia may also occur in TKI treatment, but unlike immunotherapy, TKI treatment has a higher objective response rate and does not have immunerelated potentially fatal toxicities. Exploring the efficacy of TKI maintenance in comparison with immunotherapy or the combination of these 2 drugs will have great clinical value. We expect outcomes in the following years will make the treatment of LA-NSCLC with oncogene-driven mutation more accurate and standardized.

\section{Acknowledgments}

None.

\section{Footnote}

Reporting Checklist: The authors have completed the Narrative review checklist. Available at http://dx.doi. org/10.21037/tlcr-20-722

Conflicts of Interest: All authors have completed the ICMJE uniform disclosure form (available at http://dx.doi. org/10.21037/tlcr-20-722). The authors have no conflicts of interest to declare.
Ethical Statement: The authors are accountable for all aspects of the work in ensuring that questions related to the accuracy or integrity of any part of the work are appropriately investigated and resolved.

Open Access Statement: This is an Open Access article distributed in accordance with the Creative Commons Attribution-NonCommercial-NoDerivs 4.0 International License (CC BY-NC-ND 4.0), which permits the noncommercial replication and distribution of the article with the strict proviso that no changes or edits are made and the original work is properly cited (including links to both the formal publication through the relevant DOI and the license). See: https://creativecommons.org/licenses/by-nc-nd/4.0/.

\section{References}

1. Ferlay J, Colombet M, Soerjomataram I, et al. Estimating the global cancer incidence and mortality in 2018: GLOBOCAN sources and methods. Int J Cancer 2019;144:1941-53.

2. Malapelle U, Iaccarino A, Rossi A. Cytology versus histology for programmed death-ligand 1 expression evaluation in the landscape of non-small cell lung cancer patients selection for immunotherapy. Transl Lung Cancer Res 2018;7:S221-4.

3. Stinchcombe TE, Zhang Y, Vokes EE, et al. Pooled analysis of individual patient data on concurrent chemoradiotherapy for stage III non-small-cell lung cancer in elderly patients compared with younger patients who participated in US National Cancer Institute Cooperative Group Studies. J Clin Oncol 2017;35:2885.

4. Curran WJ Jr, Paulus R, Langer CJ, et al. Sequential vs concurrent chemoradiation for stage III non-small cell lung cancer: randomized phase III trial RTOG 9410. J Natl Cancer Inst 2011;103:1452-60.

5. Aupérin A, Le Péchoux C, Rolland E, et al. Meta-Analysis of Concomitant Versus Sequential Radiochemotherapy in Locally Advanced Non-Small-Cell Lung Cancer. J Clin Oncol 2010;28:2181-90.

6. Bradley J, Hu C, Komaki R, et al. Long-term results of RTOG 0617: a randomized phase 3 comparison of standard dose versus high dose conformal chemoradiation therapy+/-cetuximab for stage III NSCLC. Int J Radiat Oncol Biol Phys 2017;99:S105.

7. Senan S, Brade A, Wang LH, et al. PROCLAIM: Randomized phase III Trial of pemetrexed-cisplatin or etoposide-cisplatin plus thoracic radiation therapy 
followed by consolidation chemotherapy in locally advanced nonsquamous non-small-cell lung cancer. J Clin Oncol 2016;34:953-62.

8. Bezjak A, Temin S, Franklin G, et al. Definitive and Adjuvant Radiotherapy in Locally Advanced Non-SmallCell Lung Cancer: American Society of Clinical Oncology Clinical Practice Guideline Endorsement of the American Society for Radiation Oncology Evidence-Based Clinical Practice Guideline. J Clin Oncol 2015;33:2100-5.

9. Bang A, Schoenfeld JD, Sun AY. PACIFIC: shifting tides in the treatment of locally advanced non-small cell lung cancer. Transl Lung Cancer Res 2019;8:S139-46.

10. Siegelin MD, Borczuk AC. Epidermal growth factor receptor mutations in lung adenocarcinoma. Laboratory Investigation 2014;94:129-37.

11. Mok TS, Wu Y-L, Thongprasert S, et al. Gefitinib or carboplatin-paclitaxel in pulmonary adenocarcinoma. N Engl J Med 2009;361:947-57.

12. Shepherd FA, Rodrigues Pereira J, Ciuleanu T, et al. Erlotinib in previously treated non-small-cell lung cancer. N Engl J Med 2005;353:123-32.

13. Solomon BJ, Mok T, Kim DW, et al. First-line crizotinib versus chemotherapy in ALK-positive lung cancer. N Engl J Med 2014;371:2167-77.

14. Ramalingam SS, Yang J, Lee CK, et al. Osimertinib as first-line treatment of EGFR mutation-positive advanced non-small-cell lung cancer. J Clin Oncol 2018;36:841-9.

15. Ramalingam SS, Vansteenkiste J, Planchard D, et al. Overall survival with osimertinib in untreated, EGFR-mutated advanced NSCLC. N Engl J Med 2020;382:41-50.

16. Akamatsu H, Kaira K, Murakami H, et al. The impact of clinical outcomes according to EGFR mutation status in patients with locally advanced lung adenocarcinoma who recieved concurrent chemoradiotherapy. Am J Clin Oncol 2014;37:144-7.

17. Hayashi H, Okamoto I, Kimura H, et al. Clinical outcomes of thoracic radiotherapy for locally advanced NSCLC with EGFR mutations or EML4-ALK rearrangement. Anticancer Res 2012;32:4533-7.

18. Ryan KJ, Skinner KE, Fernandes AW, et al. Real-world treatment patterns among patients with unresected stage III non-small-cell lung cancer. Future Oncol 2019;15:2943-53.

19. Ishihara M, Igawa S, Sasaki J, et al. Evaluation of concurrent chemoradiotherapy for locally advanced NSCLC according to EGFR mutation status. OncoL Lett 2017;14:885-90.

20. Boros A, Lacroix L, Lacas B, et al. Prognostic value of tumor mutations in radically treated locally advanced nonsmall cell lung cancer patients. Oncotarget 2017;8:25189.

21. Mitra D, Chen YH, Li R, et al. EGFR mutant locally advanced non-small cell lung cancer is at increased risk of brain metastasis. Clin Transl Radiat Oncol 2019;18:32-8.

22. Lim YJ, Chang JH, Kim HJ, et al. Superior Treatment Response and In-field Tumor Control in Epidermal Growth Factor Receptor-mutant Genotype of Stage III Nonsquamous Non-Small cell Lung Cancer Undergoing Definitive Concurrent Chemoradiotherapy. Clin Lung Cancer 2017;18:e169-78.

23. Hsia TC, Liang JA, Li CC, et al. Comparative effectiveness of concurrent chemoradiotherapy versus EGFR tyrosine kinase inhibitors for the treatment of clinical stage IIIb lung adenocarcinoma patients with mutant EGFR. J Thorac Cancer 2018;9:1398-405.

24. Zhuang HQ, Sun J, Yuan ZY, et al. Radiosensitizing effects of gefitinib at different administration times in vitro. Cancer Sci 2009;100:1520-5.

25. Das AK, Sato M, Story MD, et al. Non-small cell lung cancers with kinase domain mutations in the epidermal growth factor receptor are sensitive to ionizing radiation. Cancer Res 2006;66:9601-8.

26. Ang KK, Berkey BA, Tu X, et al. Impact of epidermal growth factor receptor expression on survival and pattern of relapse in patients with advanced head and neck carcinoma. Cancer Res 2002;62:7350-6.

27. Baumann M, Krause M, Dikomey E, et al. EGFR-targeted anti-cancer drugs in radiotherapy: preclinical evaluation of mechanisms. Radiother Oncol 2007;83:238-48.

28. Okamoto I, Takahashi T, Okamoto H, et al. Singleagent gefitinib with concurrent radiotherapy for locally advanced non-small cell lung cancer harboring mutations of the epidermal growth factor receptor. Lung Cancer 2011;72:199-204.

29. Rothschild S, Bucher SE, Bernier J, et al. Gefitinib in Combination With Irradiation With or Without Cisplatin in Patients With Inoperable Stage III Non-Small Cell Lung Cancer: A Phase I Trial. Int J Radiat Oncol Biol Phys 2011;80:126-32.

30. Xing L, Wu G, Wang L, et al. A multicenter, randomized, open-label, phase II trial of erlotinib versus etoposide plus cisplatin with concurrent radiotherapy in unresectable stage III non-small cell lung cancer (NSCLC) with epidermal growth factor receptor (EGFR) activating mutation. J Clin Oncol 2017;35:8531.

31. Akamatsu H, Harada H, Tokunaga S, et al. A Phase II Study of Gefitinib With Concurrent Thoracic 
Radiotherapy in Patients With Unresectable, Stage III Non-small-cell Lung Cancer Harboring EGFR Mutations (WJOG6911L). Clin Lung Cancer 2019;20:e25-7.

32. Choong NW, Mauer AM, Haraf DJ, et al. Phase I trial of erlotinib-based multimodality therapy for inoperable stage III non-small cell lung cancer. J Thorac Oncol 2008;3:1003-11.

33. Kelly K, Chansky K, Gaspar LE, et al. Phase III trial of maintenance gefitinib or placebo after concurrent chemoradiotherapy and docetaxel consolidation in inoperable stage III non-small-cell lung cancer: SWOG S0023. J Clin Oncol 2008;26:2450-6.

34. Ready N, Jänne PA, Bogart J, et al. Chemoradiotherapy and gefitinib in stage III non-small cell lung cancer with epidermal growth factor receptor and KRAS mutation analysis: cancer and leukemia group B (CALEB) 30106, a CALGB-stratified phase II trial. J Thorac Oncol 2010;5:1382-90.

35. Lilenbaum R, Samuels M, Wang X, et al. A Phase II Study of Induction Chemotherapy Followed by Thoracic Radiotherapy and Erlotinib in Poor-Risk Stage III Non-Small-Cell Lung Cancer: Results of CALGB 30605 (Alliance)/RTOG 0972 (NRG). J Thorac Oncol 2015;10:143-7.

36. Niho S, Ohe Y, Ishikura S, et al. Induction chemotherapy followed by gefitinib and concurrent thoracic radiotherapy for unresectable locally advanced adenocarcinoma of the lung: a multicenter feasibility study (JCOG 0402). Ann Oncol 2012;23:2253-8.

37. Komaki R, Allen PK, Wei X, et al. Adding erlotinib to chemoradiation improves overall survival but not progression-free survival in stage III non-small cell lung

Cite this article as: Jiang L, Meng X, Zhao X, Xing L, Yu J. Perspective on treatment for unresectable locally advanced non-small cell lung cancer with oncogene-driven mutation: a narrative review. Transl Lung Cancer Res 2020;9(5):2137-2144. doi: $10.21037 /$ tlcr-20-722 cancer. Int J Radiat Oncol Biol Phys 2015;92:317-24.

38. Zia NUN, Khokhar MA, Qamar S, et al. Concurrent radiotherapy and chemotherapy with erlotinib followed by maintenance erlotinib in patients with Epidermal Growth Factor Receptor mutation-positive adenocarcinoma lung. J Pak Med Assoc 2019;69:1605-9.

39. Lee Y, Han JY, Moon SH, et al. Incorporating erlotinib or irinotecan plus cisplatin into chemoradiotherapy for stage III non-small cell lung cancer according to EGFR mutation status. Cancer Res Treat 2017;49:981.

40. Hotta K, Sasaki J, Saeki S, et al. Gefitinib Combined With Standard Chemoradiotherapy in EGFR-Mutant Locally Advanced Non-Small-Cell Lung Cancer: The LOGIK0902/OLCSG0905 Intergroup Study Protocol. Clin Lung Cancer 2016;17:75-9.

41. Park K, Vansteenkiste J, Lee KH, et al. Pan-Asian adapted ESMO Clinical Practice Guidelines for the management of patients with locally-advanced unresectable non-smallcell lung cancer: a KSMO-ESMO initiative endorsed by CSCO, ISMPO, JSMO, MOS, SSO and TOS. Ann Oncol 2020;31:191-201.

42. Tan WL, Chua KLM, Lin CC, et al. Asian Thoracic Oncology Research Group Expert Consensus Statement on Optimal Management of Stage III NSCLC. J Thorac Oncol 2020;15:324-43.

43. Santaniello A, Napolitano F, Servetto A, et al. Tumour Microenvironment and Immune Evasion in EGFR Addicted NSCLC: Hurdles and Possibilities. Cancers (Basel) 2019;11.

(English Language Editor: J. Gray) 